\title{
Édition et analyse de la Remontrance à Monsieur par un Français de qualité (1631)
}

\section{Giuliano Ferreti et Francis Goyet}

\section{OpenEdition}

1 Journals

Édition électronique

URL : https://journals.openedition.org/rhetorique/1197

DOI : 10.4000/rhetorique. 1197

ISSN : 2270-6909

Éditeur

UGA Éditions/Université Grenoble Alpes

Édition imprimée

ISBN : 978-2-37747-325-0

Référence électronique

Giuliano Ferreti et Francis Goyet, «Édition et analyse de la Remontrance à Monsieur par un Français de qualité (1631) », Exercices de rhétorique [En ligne], 17 | 2021, mis en ligne le 25 novembre 2021,

consulté le 29 novembre 2021. URL : http://journals.openedition.org/rhetorique/1197 ; DOI : https:// doi.org/10.4000/rhetorique.1197

Ce document a été généré automatiquement le 29 novembre 2021.

\section{(c) (7) ()}

Les contenus de la revue Exercices de rhétorique sont mis à disposition selon les termes de la Licence Creative Commons Attribution - Pas d'Utilisation Commerciale - Partage dans les Mêmes Conditions 4.0 International. 


\title{
Édition et analyse de la Remontrance à Monsieur par un Français de qualité (1631)
}

\author{
Giuliano Ferreti et Francis Goyet
}

\section{Édition - Remontrance à Monsieur par un Français de qualité (1631), par Richelieu ou Jean Sirmond ( ?)}

Nous reproduisons ici, dans sa quasi-intégralité, la Remonstrance à Monsieur par un François de qualité (1631), anonyme et traditionnellement attribuée à Richelieu, que nous faisons suivre d'une analyse historique puis rhétorique. Cette double analyse reprend, avec la très aimable autorisation des éditions Classiques Garnier, la contribution suivante : Giuliano Ferretti et Francis Goyet, « La Remontrance à Monsieur (1631) : une remontrance irrégulière ? ", dans Ullrich Langer et PaulAlexis Mellet dir., Les Remontrances (Europe, $\mathrm{XVI}^{e}$-XVIII ${ }^{e}$ siècle). Textes et commentaires, Paris, Classiques Garnier, 2021, p. 249-263.

Plaquette anonyme, et sans lieu d'édition. Nous reproduisons quasi-intégralement l'exemplaire de la Bibliothèque Municipale de Lyon (cote 315728), visible sur Gallica. Graphies modernisées, accords grammaticaux régularisés; remplacement de :) par ): et de l'esperluette par et ; casse, ponctuation et alinéas conservés ; suppressions signalées. Nous introduisons cinq intertitres en les faisant précéder d'une ligne blanche. Toutes les indications entre crochets droits sont nôtres, dont l'indication de la pagination d'origine, de la page 3 à la page 44 .

REMONTRANCE À MONSIEUR, Par un Français de qualité. M. DC. XXXI. 


\section{MONSEIGNEUR,}

1 [3] Les monstres d'envie, d'avarice, et d'ambition qui vous environnent, empêcheront je m'assure que ce Discours tout plein de liberté, ne pénètre jusques à vous. Je ne doute nullement, qu'outre les grandes connaissances que les Princes tiennent de Dieu, vous n'ayez des lumières naturelles si puissantes, que même dès votre enfance vos raisons, plus que pertinentes, faisaient espérer à toute la France des effets d'esprit et de courage tout à [4] fait extraordinaires: Les dispositions, en sont encore en vous, et quelques soins que prenne la flatterie, qui ne vous a point abandonné jusques ici, votre jugement détrompé se peut joindre aux justes ressentiments que doivent avoir tous les gens de bien de votre éloignement de la Cour, et des sujets qui séparent les volontés du Roi et les vôtres au grand avantage des ennemis de cette Couronne. La naissance qui vous est commune à tous deux, l'inclination à la vertu, la nourriture presque pareille, et l'union de vos fortunes, sont les seuls ressorts qui doivent faire jouer votre bonne intelligence. Que si jusques ici les défauts de vos Ministres en ont changé les mouvements, la voix de Dieu qui vous [5] rappelle, le ressentiment d'un Roi qui vous veut traiter en frère et comme fils, les souffrances d'un peuple qui succombe à tant de misères, dont le bras de Dieu l'a châtié depuis quelques années, doivent contraindre vos intérêts, déguisés par leur malice, de reconnaître que votre gloire et la réputation de la France dépendent de votre retour et de la paix. Le Roi la souhaite, et elle sera toujours procurée à votre contentement par ceux qui gouvernent sous lui : car quelque créance que l'on vous veuille donner de leurs intentions, elles ne peuvent être mauvaises, si l'on considère leurs actions passées, leurs efforts pour en former de plus glorieuses, et leurs desseins qui eussent déjà fait dominer la France à tout le monde, [6] si la fortune eût aidé leurs désirs, et la bonne conduite de notre Prince.

2 Vous n'ignorez pas, Monseigneur, que le Cardinal de Richelieu, que vous faites le Ministre de vos divisions, ne soit tout plein de courage et d'adresse : mais vous vous plaignez qu'il destine pour votre ruine les bonnes parties qu'il devrait employer au bien de ce Royaume (pardonnez-moi si je parle de la sorte, la vertu a de si fortes apparences, que je l'adore même en mes ennemis)[.] [L]'éclat de sa fortune et l'espérance de la partager, ne me feront jamais flatter la vérité : si ce Discours lui tombe dans les mains, il n'y reconnaitra que la liberté d'un Français qui cache son nom pour n'avoir d'autre intérêt que [7] celui de l'État. Voyons ce qu'il a fait pour vous.

Vous vous plaignez qu'il vous recule des affaires, et que tout ce qui environne le Roi, ne lui laisse pas seulement la liberté des sens : nous savons que tous ceux qui le veulent, y rencontrent l'accès très facile, et qu'il écoute plus que Prince du monde les plaintes de ses sujets : tous ceux de la Cour lui parlent tous les jours, et vous-même avez eu d'assez longs entretiens avec lui pour rencontrer les occasions de lui faire connaître vos mécontentements, et les grands desseins du Cardinal. Même pardonnez-moi si je dis que vous avez eu tort de ne le pas faire, si tant est que vous les ayez reconnus armés contre le Roi, la [8] Reine votre mère et votre personne: mais je crois que vous n'en avez eu les parfaites connaissances que depuis votre départ de la Cour, que l'on vous conseilla par de malicieuses appréhensions que vous en donna votre Chancelier, afin de vous éloigner des lieux, où vous pouviez découvrir le contraire de ce qu'il vous disait. 


\section{[I. Narration du passé1 : années 1625/26 à 1630]}

Retournez-vous, et regardez le passé : Savez-vous pas que dès la naissance de sa fortune, il vous trouva éloigné des affaires, dans quelques défiances, et sans apanage? II fit tout ce qui lui fut possible pour vous faire vivre en frère avec le Roi, qui vous donnait souvent la moitié de son lit, afin de s'acquérir votre confidence, et de détourner [9] les mauvais conseils que ceux de l'âge que vous aviez pour lors sont capables de recevoir. Le Colonel homme hardi et ambitieux fut celui qui ruina ces bonnes intentions, porté de fantaisies qui lui étaient fort considérables pour lors. $\mathrm{Ne}$ doutez pas, Monseigneur, que les desseins de ceux qui ont l'honneur d'approcher les grands Princes, ne montent toujours, et qu'après s'être donné créance auprès de leurs maîtres, ils ne les portent à tout entreprendre : Il y en a peu qui le fassent pour avancer leur gloire, mais pour leur témoigner qu'ils leur sont tout à fait nécessaires, et de là s'acquérir une autorité dans leurs actions dont bien souvent ils abusent. L'histoire que vous savez très bien, vous a fait [10] connaître le secret d'une si mauvaise maxime; et quand il vous plaira de conférer sans passion avec votre jugement, il vous dira que le Colonel ne travaillait pas tant à votre établissement, qu'au sien particulier, par les factions qu'il formait dedans la France et hors du Royaume.

Ce furent les sujets qui obligèrent le Roi à le faire arrêter avant que de s'en plaindre à vous dont la passion avait été abusée par les artifices d'un homme si malicieux : L'on connut bien qu'il vous flattait de belles espérances, mais que ce n'était que pour assurer les siennes, et qu'il voulait vous faire servir de jouet à la fortune d'un autre. Le Roi ne vous en traita pas plus mal, au contraire dès lors il vous donna [11] grand apanage, vous maria avec une grande Princesse pleine de vertus et de biens, et fit tout ce qu'il pût pour vous dégager des reliques des desseins du Colonel, pour embrasser l'intérêt de l'État qui ne peut être que le vôtre. [...]

$6 \quad[\ldots 12]$ Et vous direz que le Cardinal vous veut reculer des affaires, et qu'il nourrit des défiances dans l'esprit du Roi pour établir sa puissance, et pour vous détruire? Si vous eussiez alors écouté la vérité de ses conseils, si vous eussiez déféré aux volontés du Roi qui vous [13] conjurait d'oublier toutes choses, de reconnaitre à quoi l'on se voulait servir de vous, que l'on abusait de vos bontés, et que tout de bon vous eussiez embrassé la part dans le gouvernement des affaires telle que l'on vous l'offrait, vous ne seriez pas hors de France environné de factieux, qui ne pensent qu'à vous détruire, et à vous détourner de ce que vous devez à vous-même: Mais vous demeurâtes dans vos premières opinions, et les créatures de vos ennemis couverts qui restaient auprès de vous, ne cessèrent de vous cajoler pour vous armer pour la défense des vôtres (ce disaient-ils) et sous ce prétexte vous faisaient écouter des propositions que leurs esprits méchants et ulcérés vous [14] rendaient faciles pour le salut du Colonel, et qui tacheront votre réputation dans les siècles, si tout le monde ne croit (comme de fait) que vous n'en vouliez pas au Roi, mais au Cardinal, qui était l'auteur de vos mécontentements : quoi que ce soit crime d'attenter aux Ministres de l'État, qui sont comme les organes de la Monarchie.

7 Ces gens, Monseigneur, qui vous embarrassèrent dans ce tracas, ne vous disaient pas que la vanité de vos efforts vous décréditerait et dans la France et chez les Étrangers: vous en sentirez les effets si vous continuez dans le dessein d'armer contre le Roi (ce que Dieu ne veuille) les Princes et la Noblesse vivant dans [15] la créance que vous êtes pour émouvoir de grands troubles, et pour en terminer bien peu. Je ne dis pas que vous 
n'ayez en vous tout ce qui peut former un sage et généreux Prince : mais vos actions ont fait connaître à tout le monde que vous croyez trop le conseil de vos Ministres (ce que vous blâmez en notre Roi): encore si vous faisiez des élections pareilles aux siennes, je ne serais en peine que d'écrire les combats que vous auriez gagnés sur les ennemis de l'État, et la prise de tant de villes, où votre naissance et votre courage vous donnent droit.

[... 18] Ce sont ces bons serviteurs que vous avez auprès de vous, à qui l'adresse et le courage ont crû depuis ce temps-là, par les conseils que leur inspire le sieur de Monsigot, qui cherchait à quelque prix que ce fût, de débiter les notions qu'il s'était acquises pendant qu'il était Secrétaire du Connétable de Luynes: avant ce choix ils faisaient tout si à contretemps que la plupart des gens de bien disaient tout haut qu'ils vous abusaient, qu'ils étaient pensionnaires du Cardinal, et les plus déliés : que cela ne pouvait être, vu les lourderies qu'ils faisaient, et qu'il était trop adroit pour ne leur pas prescrire des fourbes plus spécieuses. Je [19] vous prie Monseigneur, que je vous en remarque quelques-unes.

Sont-ce pas ces Messieurs qui vous firent quitter le siège de la Rochelle et qui vous ramenèrent à Paris, vous faisant valoir beaucoup d'y gouverner en l'absence du Roi ? Sont-ce pas eux qui vous y entretenaient dans le plaisir, tandis que le Roi suait dans les travaux d'un siège ? Sont-ce pas ces fidèles Conseillers, qui vous laissèrent embarrasser d'amour au lieu de vous mener dans l'école de la guerre, qui est un vrai métier d'un Prince? Mais je vous prie, comme quoi vous ont-ils conduit dans cette action? S'ils n'ont été les instruments de la ruine de Monsieur de Mantoue, au moins ils en ont eu le dessein. Ils ont enduré que vous ayez vu [20] emprisonner deux grandes Princesses, toutes deux vos parentes, et l'une votre Maîtresse, sans crime que pour être aimée de vous; vous le souffrîtes, quoique tout le monde se préparât de vous assister dans une boutade d'amoureux : mais vous vous conseillâtes plutôt à eux qu'à votre passion; et ceux qui vous procurèrent ce déplaisir par leurs conseils, ce sont ceux-là pour la défense desquels vous vous armez maintenant. Eussiez-vous pas trouvé dans Paris vingt mille jeunes gens qui pouvaient avec vous l'enlever de sa prison et l'épouser sans déplaire au Roi qui n'y répugna jamais ? vous eussiez offensé la Reine votre mère, où est la Loi qui nous oblige d'obéir à nos parents en leurs passions? Le Roi qui [21] vous doit servir de père aussi bien qu'il est votre frère, n'eût pas fait un autre choix pour luimême, comme il l'a témoigné plusieurs fois : mais la corruption [qui] se glissa dans le cœur des vôtres, et la crainte qu'ils avaient de piquer la Reine dans ses intérêts, éludèrent toutes les résolutions de votre amour, qu'ils réservaient au temps pour lui donner le remède qu'il a trouvé: Et puis ils destinaient votre fantaisie pour être l'instrument de votre réconciliation avec la Reine, qui vous abandonnait le Cardinal si vous vous éloigniez de vos autres désirs. Pensez-vous que Dieu ne punisse pas les serments des amoureux et qu'il ne se souvienne pas que dans l'hôtel de S. Paul vous le reniâtes pour tel, si jamais vous changiez de [22] volonté ? Et cependant vous écoutez d'autres partis que l'on vous propose, et vous témoignez par vos froideurs que vous ne l'aimez plus. Vous ne vous souvenez plus des larmes qu'elle a jetées tant de fois auprès de vous pour les sujets qui vous touchaient et l'un et l'autre: Vous avez oublié la mort de sa Tante dont vos intérêts furent la cause, et vous souffrez encore que celle que vous disiez gouverner si absolument dans votre âme, vive dans un cloître parmi des Religieuses, qui est une vie bien éloignée de celle que vous lui deviez procurer. 
10 Tout cela serait peu de chose si vous ne connaissiez que la Reine votre mère toute pleine de désirs de vengeance contre la maison de Mantoue a sans cesse traversé ses [23] affaires pour en perdre le chef, et pour vous détourner d'épouser la fille d'un Prince ruiné ? Les diverses négociations que l'on a faites auprès des Princes armés pour le détruire, les lettres surprises pendant la maladie du Roi, sans parler de beaucoup d'autres choses, que j'aime mieux taire que publier; seront d'assez puissants témoignages aux siècles à venir de ce que j'écris : comme si la Princesse Marie était cette fille de Nisus qui devait arracher le poil fatal de son père, et lui enlever quant et quant son État pour le sacrifier aux désirs d'un Prince qui ne s'en soucie plus. Le pillage de Mantoue et de tant d'autres places, les maux soufferts par tant de milliers de personnes, les meurtres et les violements qui s'en sont [24] ensuivis, sont-ce pas les effets de sa passion et de la vôtre?

11 Et puis vos Ministres vous veulent relever d'honneur au-delà de ce qui fut jamais! Ce fut cette raison qui vous fit refuser le commandement de l'Armée d'Italie de peur d'irriter la Reine, à laquelle ils avaient jà fait espérer cette réconciliation. Mais pourtant que firent-ils pour venger vos mécontentements, quand vous vous retirâtes en Lorraine la première fois? Ce ne fut pas avant le secours donné à l'Italie, ni devant la conquête du Languedoc, pendant laquelle les maux pressants, et les cœurs élevés vous donnaient beau jeu d'entreprendre pour vous faire écouter, l'on vous eût fait revenir avec toutes les satisfactions que vous eussiez souhaitées : mais [25] je m'assure qu'ils furent si bons Français qu'ils ne voulurent traverser la prospérité des armes du Roi, et qu'ils voulurent attendre la paix, afin de ne donner que les appréhensions du mal. On le connut très bien, car ils ne firent la guerre que par lettres et par défiances, et trouvèrent votre réunion et la leur dans un supplément d'apanage, quelques récompenses, et des assurances qui adoucirent leurs craintes, et qui vous apaisèrent, puisque vous avez toujours vécu jusques ici plus dans leurs intérêts que dans les vôtres.

12 Voilà, Monseigneur, quelle a été la conduite de votre conseil pendant qu'il agissait par ses seuls mouvements: Jugez si ces traverses d'esprit qu'ils vous ont données [26] durant quatre ou cinq années, vous ont avancé davantage dans les affaires et dans la bonne grâce du Roi, et vous ont fait acquérir grande créance et dans la Cour et dans le reste de la France. Détrompez votre raison, et vous assurez que les résolutions qu'ils ont prises depuis avec d'autres plus adroits et plus malicieux qu'ils ne sont pas, ne relèveront pas davantage votre gloire.

\section{[II. Narration du présent ${ }^{2}$, à partir de la Journée des Dupes, le 11-12 novembre 1630]}

13 Ils vous ont uni avec la Reine, j'avoue que c'est un acte de piété de réduire un fils dans l'obéissance de sa mère, pourvu que ce soit à bonne fin : Car il faudrait être bien simple pour ne savoir pas qu'on peut aussi bien offenser Dieu en obéissant à une mère qui commanderait chose mauvaise, comme en lui désobéissant en [27] chose bonne, où Dieu lui donne autorité de commandement. Mais quand je considère la fin de leur dessein, je m'étonne qu'ils osent gourmander le Roi dans ses élections, qu'ils le veuillent forcer de se servir d'eux, et de reconnaître les grands services du Cardinal par une insigne ingratitude, comme s'ils avaient affaire à un Prince privé de sens, sans connaissance du vrai et du faux, et qui ne peut mettre différence entre des personnes piquées d'un zèle 
très capable, et d'une louable ambition; et des gens qui se trouvent bien empêchés à régler leurs familles et leurs vies.

Voilà de jolis Conseillers d'État, ils n'avaient garde de porter eux-mêmes leurs remontrances, et de sonner le combat: ils se [28] contentent d'en ordonner, et choisissent pour porter cette parole la Reine votre mère, qui les assurait par le Garde des Sceaux et par le Maréchal son frère de la perte du Cardinal, par la forte créance qu'elle s'était donnée dans l'esprit du Roi, qu'elle se promettait de ranger au point qu'elle désirerait.

15 Mais voyez, Monseigneur, où vous porte la vanité de vos espérances, fondées sur les apparences de si faibles conseils. Vous voyez que le Roi n'écoute pas la calomnie ; qu'il aime davantage, qu'il combat de raison, enfin qu'il trouve tant de sûretés en la fidélité du Cardinal, qu'il souffre plutôt l'éloignement de sa mère que la perte d'un homme tant utile au bien de ses affaires et de son État. [29] Vous en témoignâtes de grands ressentiments, et de fait vous y étiez obligé puisque vous étiez de la partie : mais vous ne deviez pas ensuite jurer amitié au Cardinal, et souffrir que les vôtres s'engageassent par les grandes sommes et les charges qu'ils reçurent du Roi pour arrhe de l'avoir trompé, ou de l'avoir été eux-mêmes. C'est ce qui les marquera à jamais de légèreté, et d'une forte avarice, et ce qui fera condamner votre séjour hors de la France quand vous en auriez tous les sujets du monde. Et ce sont les meilleures raisons que puisse avoir le Cardinal pour opposer aux calomnies qu'ils lui imputent tous les jours.

Cela n'est rien, Monseigneur, si vous ne considérez qu'ils ont [30] voulu piper, et vous et la Reine votre mère. Sait-on pas que les Marillac ont encore les cœurs tout enflammés des feux de la sainte Ligue, et que le Garde des Sceaux est trop plein de conscience pour ne demeurer pas dans l'observance d'un serment qu'il signa de son sang? connaît-on pas l'opiniâtreté de ses résolutions qu'il ne changea jamais, et l'ambition de son frère qui ne regardait rien moins qu'une épée de Connétable, dès lors que l'on lui fit l'honneur de le charger d'un bâton de Maréchal de France? Pour y parvenir quels ressorts font-ils jouer, ils procurent votre réconciliation avec la Reine, à laquelle ils montrent le Cardinal comme un homme qui se voulait élever sur les ruines de ses enfants, elle qui [31] avait tant de fois dit qu'il ne pouvait faillir : ils veulent lui faire connaitre que par une ingratitude nonpareille il ne lui rend que des devoirs déguisés, et qu'il lui cache tout ce qu'il y a de fin dans le Conseil qui n'est plus que dans le secret du Roi et dans le sien; qu'il détourne l'obéissance du Roi et cet amour filial qu'il lui avait toujours témoigné, et ne sortent jamais de ces discours d'ingratitude, comme les plus sensibles moyens qui les peuvent conduire au point où ils aspirent : que si le cœur lui en dit, les semences d'amour sont encore assez fortes dans l'esprit du Roi pour se laisser persuader à ce qu'elle lui voudra inspirer: que le Cardinal ne s'y maintient que par elle-même : que ce sont les uniques voies [32] pour vous acquérir pour son salut qui ne pouvait plus être qu'en vous, puisque la faiblesse d'une femme était trop peu de chose pour opposer aux pernicieux desseins qu'ils disaient qu'il faisait déjà éclater contre l'État dont il usurpait toutes les forces.

Ils flattaient bien encore davantage ses espérances, que c'était le moyen de vous dégager d'avec la Princesse Marie, et de vous ranger dans l'alliance de Florence, ce qu'ils savaient être son principal désir, et ce qui a plutôt armé sa résolution contre un homme qu'elle n'eût jamais abandonné après l'avoir si hautement protégé et lui l'avoir si dignement servie. 
, Monseigneur, ils vous ont entretenu de discours presque tous pareils, et vous on proposé [33] ce que vos ministres briguent il y a longtemps, à savoir le maniement des affaires : et pour y atteindre ils dressent force pratiques dehors et dedans le Royaume, forment de grands desseins, qui ne se peuvent couvrir étant communiqués à tant de personnes qui ont été leur perte, et qui vous ruineront de réputation, si vous ne vous rendez dans les respects que vous devez au Roi, lequel sera toujours prêt de vous satisfaire dans les torts que vous prétendez vous être faits, si la justice et le bien de son État le lui conseillent.

\section{[III. Objection et réponse à l'objection : la réfutation $\left.{ }^{3}\right]$}

19 L'on sait que vous vouliez dire que vous ne pouvez avoir la créance que le Cardinal n'en veuille au Roi et à l'État. Pour tenir [34] quelques gouvernements et quelques charges, ce n'est pas arracher les fleurons d'une Couronne si puissante; et une compagnie de gardes armée pour le défendre de tant d'attentats que l'on a fait contre sa vie, ne suffira pas pour conquérir un Royaume : il n'est pas croyable que tous les Français qu'il oblige par ses libéralités, et auxquels il a commandé dans les emplois, voulussent l'assister contre leur Roi, ils seraient les premiers qui conjureraient à sa ruine ; et quant à moi je ne puis arrêter ma plume sur un sujet si horrible, si criminel, et éloigné d'apparences.

Le Cardinal ne fait pas plus et ne possède pas davantage que les favoris des Rois qui ont [35] précédé le nôtre : si vous voulez obliger le Roi à prendre le soin entier de son État, vous voulez ce qui ne fut jamais fait (ce qui serait capable d'abréger la vie du plus robuste homme du monde) et enfin ce que Dieu ne demande pas d'eux: ains au contraire, agissant par ses causes secondes, il leur apprend à se servir de leurs sujets en leur ministère. Le défunt Roi le plus laborieux des Princes qui furent jamais, n'avait-il pas des ministres qui partageaient les affaires? Et vous-même, Monseigneur, prenezvous tous les soins de votre maison et de votre fortune? Vos Ministres en seraient très fâchés, et n'auraient pas de si grandes sommes aux intérêts chez les Partisans. Que si dans la [36] conduite particulière des affaires quelque chose vous semble n'aller pas, comme la raison voudrait, laissez-nous seulement en paix, et vous verrez que le Cardinal sera le premier à seconder de son assistance vos louables intentions auprès de sa Majesté. Croyez-vous qu'après avoir fait régner le Roi si absolument, qu'après s'être réconcilié avec vous et la Reine Mère, qu'après avoir procuré la paix à ce Royaume, il ne fasse pas tout ce qui lui sera possible pour acquérir les bénédictions du peuple, qu'il n'a pu soulager jusques ici, vu les désordres qui ont brouillé cette Monarchie, et qui nous menacent encore si vous ne retournez?

\section{[IV. Narration du futur, avec appel à une passion, la crainte $\left.{ }^{4}\right]$}

[37] Reconnaissez, Monseigneur, que vous ne pouvez vivre plus glorieux qu'en France, où tant de peuples vous obéissent, où le Roi vous chérit, où chacun s'efforce à vous faire l'honneur qui est dû à votre naissance: Avouez que vous souffrez partout ailleurs, et que les effets sont bien éloignés des promesses étrangères : Chacun considère ce que vous pouvez de vous-même, et si vous continuez, vous ferez que l'on s'étonnera de votre impuissance. Ceux dont vous implorez l'assistance[,] s'ils sont étrangers, ont assez d'affaires chez eux, sans vous assister dans une boutade qui doit finir à leur désavantage : s'ils sont Français, ils ne contribueront à vos desseins que des civilités et [38] des offres, dont vous ne verrez jamais les effets, vu le bon ordre que l'on y met tous

Exercices de rhétorique, 17 | 2021 
les jours, et l'impuissance où ils se trouvent pour avoir autrefois mal ménagé leurs biens et leurs fortunes durant la minorité du Roi. Ce qui vous restera, seront la plupart gens déterminés, esprits ulcérés et mal contents, les autres pour se faire connaître, et d'autres pour vivre dans la liberté des armes civiles. Quelle discipline pourrez-vous établir parmi des personnes de cette sorte? Vous verrez que vous aurez plus de Capitaines que de soldats, et vous repentirez de n'avoir pas écouté le Conseil du Roi, qui vous conjure de retourner il y a si longtemps, non pas par la terreur de vos [39] armes, mais par des considérations d'amour qui touchent tout le monde.

Vous ne savez que trop bien le bon ordre établi par toutes les frontières que vous pouvez menacer dans les provinces où il reste de vieilles plaies qui saignent encore, l'obéissance des Princes et l'amour de la Noblesse et du peuple qui maudira vos entreprises des mêmes lèvres dont il bénit le Roi. Vous verrez que nous vivons en bonne intelligence avec le Duc de Savoie : les Anglais blâment hautement vos desseins, et disent qu'ils ne veulent point aider les ressentiments d'une Mère contre un fils : nous sommes en paix avec l'Espagne et l'Empereur, et quoiqu'ils vous considèrent pour [40] l'exécution du Traité de Mantoue, ils sont si pressés d'ailleurs que l'on les forcera d'y consentir quand bien vous n'y contribueriez pas. Et puis encore ne ferez-vous pas la guerre toute votre vie, il faut que le malheur de l'un et de l'autre parti ramène la paix. Croyez-vous que celui du Roi puisse succomber avec les assistances de Dieu, de la Justice et de toutes ses puissances? Quand bien toute l'Europe se joindrait à vous, vous laisseriez à conquérir à ceux qui viendraient après vous. Aussi de vouloir croire que le Roi vous accorde dans un Traité tout ce que vous voulez, c'est tout ce qu'il pourrait faire s'il était votre sujet comme vous êtes le sien.

Le Cardinal, qui occupe toutes [41] ses affections, sera-[t-]il à votre gré d'humeur à se défaire de la place qu'il tient dans l'État pour se retirer ou à Rome ou dans quelquesunes de ses maisons? Pardonnez-moi, Monseigneur, si je vous dis que vous auriez une mauvaise créance après avoir connu son courage : étant résolu de perdre plutôt la vie que d'abandonner son Maître, il s'estimerait indigne de le voir seulement jamais, s'il avait commis cette lâcheté.

\section{[V. Exhortation finale ${ }^{5}$}

Retournez sans traiter, et touché du Ciel venez-vous remettre dans les bras du Roi, où vous finirez tous les desseins qui hasardent votre vie, et votre fortune, et qui troublent le repos de la France. Souvenez-vous de Charles [42] Duc de Lorraine, oncle de Louis V et dernier mâle de la seconde lignée de nos Rois (encore que les cœurs des Français ne soient pas capables des mauvaises résolutions de ceux des siècles passés) : n'autorisez plus les injures que l'on fait courir par la France sous votre nom, au mépris de la personne du Roi, de l'autorité Royale et des ministres d'icelle : ne cherchez plus des places de sûreté que dans le cœur du Roi que vous toucherez d'un repentir fait de si bonne grâce : éloignez-vous des mauvais conseils; et si pour quelques considérations, vous chérissez encore les ministres d'iceux, cherchez leur assurance dans votre salut, et croyez qu'ils [43] sont trop peu de chose pour servir d'instrument aux vengeances d'un grand Roi. Ce sera lors que vous remettrez les intérêts de la Reine votre mère au point que vous les voudrez porter raisonnablement, et que vous étonnerez tant d'esprits séditieux qui vous entretiennent dans les troubles. 
Joignez le conseil du Cardinal aux bonnes parties que votre naissance vous a données, et que votre cœur vous a acquises; et je m'assure que vous n'aurez pas longtemps vécu de cette sorte, que vous ne serez plus capable d'aucuns mauvais désirs contre son établissement, et que vous n'aurez plus de pensée que de porter les armes du Roi, et [44] votre gloire aux endroits où l'on appréhende que vous les portiez : ce que je vous persuade, ce que Dieu vous inspire, et ce que tous les bons Français attendent d'un Prince Chrétien, qui devez vivre dans l'obéissance du Roi puisque la Loi vous y a soumis.

\section{Analyse de la Remontrance}

\section{Introduction : présentation générale}

Suite au "Grand Orage» que fut la Journée des Dupes (novembre 1630), Gaston d'Orléans, frère de Louis XIII et héritier du trône, rompt ouvertement avec la cour fin janvier 1631 et se rallie à Marie de Médicis et à son parti, ou ce qu'il en restait, catalysant autour de lui une partie de l'aristocratie. En février, il se retire à Orléans où il organise ses forces puis, indigné par l'éloignement de sa mère à Compiègne, il part vers le sud le 13 mars, passe par la Bourgogne et s'arrête enfin à la cour de Nancy. Le 28 mars, il avait quitté le royaume. Le départ de l'héritier du trône étant un danger politique majeur, cela obligeait la monarchie à faire de son mieux pour faire revenir l'illustre fugitif. La difficulté de cette situation se mesure au nombre de lettres, relations, déclarations, injonctions, officielles ou non, que les protagonistes firent circuler pour justifier leurs actions. Plusieurs libelles furent également publiés par le cabinet de presse de Richelieu, dont notre texte.

Celui-ci s'intitule : Remonstrance à Monsieur par un François de qualité, anonyme, s. 1., 1631 (désormais "la Remontrance ", que nous citerons en graphies modernisées). Suivit une réfutation, anonyme mais attribuable à Matthieu de Morgues : Charitable Remonstrance de Caton chrestien à [...] Richelieu sur ses actions, et quatre libelles diffamatoires, faicts par luy ou ses escriuains ${ }^{6}$. Nous désignerons ce second texte (cité en graphies anciennes) par un simple « Morgues».

Le titre de "remontrance ", unique dans la production du cabinet de Richelieu, pose problème à Morgues (p.48): "vostre Remonstrance [...] deuroit estre remplie de respect (si vostre intention estoit que Monsieur en fist son profit)». C'est expliciter deux règles: le respect et l'intention, c'est-à-dire 'l'humilité' de l'orateur (les « humbles» remontrances) et la visée d'« instruction» (Morgues, p. 29). Notre propos sera de situer la Remontrance par rapport à ces deux règles, et de voir si elles sont valides. Dans un contexte de crise majeure, on peut penser a priori que l'idée de régularité est problématique.

Cette brève présentation ne serait pas complète sans éclaircissement sur l'auteur probable de la Remontrance. Celle-ci ne porte ni nom d'éditeur ni marques typographiques permettant d'identifier l'atelier de fabrication. Selon Maximin Deloche ${ }^{7}$, sa haute qualité éditoriale prouverait qu'il s'agit d'un document officiel. Plusieurs exemplaires sont conservés dans les bibliothèques françaises, dont des parisiennes et cinq à la seule $\mathrm{BnF}^{8}$. En 1632 parut une autre édition (s. l., s. n.), dont ne subsiste qu'un seul exemplaire ${ }^{9}$. Puis Paul Hay du Chastelet la reprit dans son Recueil de diverses pièces pour servir à l'histoire (éditions 1634, 1635, 1639, 1640, 1643, etc.). Or ce 
Recueil réunissait les pamphlets faisant l'éloge de Richelieu, et celui-ci participa à leur sélection.

Selon Deloche (op.cit., p. 465), la Remontrance fut attribuée à Richelieu par la voix publique, attribution explicite, dès 1631, dans le titre de Morgues. Les différents spécialistes, Lelong, Barbier et surtout Donald A. Bailey ${ }^{10}$ sont du même avis, mais l'affaire est plus complexe. Richelieu, comme tout haut prélat, employait plusieurs écrivains, si bien que son œuvre multiforme présente des styles si variés qu'il s'avère difficile de l'attribuer à un seul homme. Morgues en est un témoin précieux, lui-même ayant été membre du cabinet de Richelieu, avant de choisir la cause de la reine mère. Certes, s'adressant à Richelieu il précise d'emblée « qu'on croit qu'elle [la Remontrance] est de vostre stile"(p.34). Mais il remarque à propos du mot "favoris» de la Remontrance (à sa p. 34, ici à III, \$ 20) : « vostre discours est mauvais, lorsque vous vous appellez fauory", et il invite Richelieu à s'en plaindre à l'auteur de la pièce : "Si vous n'avez faict cet escrit, reprenez hardiment ce sot Escrivain, qui vous appelle fauory, qui est aujourd'huy le nom des chiens de couchette ${ }^{11} »$ (Morgues, p. 92-93).

31 Qui a donc écrit la Remontrance ? En 1630, les écrivains de talent au service du cardinal n'étaient pas nombreux. Suite à la Journée des Dupes, Richelieu commandita divers ouvrages aux fidèles Louis de Guron et Hay du Chastelet: mais ceux-ci étaient des plumitifs de second ordre. À cette époque, seul Jean Sirmond se détachait de ce groupe. Historien et littéraire raffiné, écrivain de race, il avait su se faire apprécier par le cardinal qui le chargea d'écrire plusieurs pièces dont le Coup d'Etat de Louis XIII et $L a$ defense du Roy et de ses ministres, tous parus en 1631. Morgues en estima la plume et précisa même que, pour le Coup d'Etat, Richelieu avait attribué à Sirmond 12000 livres $^{12}$, somme énorme, à la mesure de l'enjeu. La qualité littéraire de la Remontrance ainsi que l'importance de son destinataire nous font donc penser que Jean Sirmond est aussi l'auteur de notre texte. Celui-ci travailla probablement sur le canevas préparé par Richelieu et dut en relier les notes hâtives et les passages clefs chers au cardinal, selon la pratique de son cabinet de presse.

\section{Analyse historique : « respect » et hiérarchie}

La Remontrance à Monsieur renvoie à un enjeu politique fondamental au XVII ${ }^{\mathrm{e}}$ siècle, le triomphe de la monarchie absolue, qui s'est manifesté par la Journée des Dupes et par le rôle de ses trois protagonistes : le roi, son frère et sa mère. C'est sur ce fond qu'agit le principal ministre qui, bien qu'incontournable, joue un rôle subordonné sur le plan hiérarchique. Contrairement à ce qu'une longue vulgate historiographique a affirmé, Richelieu n'est que le second dans une stratégie menée avec grande habileté par Louis XIII contre Gaston d'Orléans et Marie de Médicis.

33 Nous allons regarder successivement la place de Monsieur, celle du roi et enfin celle de Richelieu. Parler de place est en effet une manière de rendre compte du manque de « respect» que Morgues reproche à la Remontrance. Tout le problème est que le « coup d'État » venu d'en haut a déstabilisé les places de chacun, y compris celle du ministre.

\section{La place de Monsieur}

Comme frère et héritier du roi, Gaston d'Orléans est une personnalité forte et faible à la fois. Dans une monarchie stable, il est acculé à un rôle subordonné, biologique et de figuration, relevant des décisions du roi qui en redoute par définition l'initiative 
politique. Mais dans une monarchie instable confrontée à une opposition vivace, nobiliaire notamment, Monsieur dispose d'un espace allant de la défense de ses marques d'honneur à l'affirmation de son indépendance politique, pour reprendre les analyses de Jean-Marie Constant et Pierre Gatulle. Leur étude de la dimension privée et publique de Monsieur à la lumière de son statut 'royal' permet de mieux comprendre la trajectoire politique du prince, interprétée traditionnellement comme confuse et contradictoire ${ }^{13}$.

Relégué à un rôle subalterne par son frère et par sa mère, Monsieur subissait en effet les « vexations » (Constant) liées à son statut et rechercha une forme d'autonomie. Son premier mariage (avec Mademoiselle de Bourbon-Montpensier), décidé par Marie de Médicis et Louis XIII, puis le conflit de la Journée des Dupes le poussèrent à manifester sa volonté d'indépendance. Celle-ci se concrétisa par trois sorties du royaume, en 1631 mais déjà en 1629 et de nouveau en 1632, ainsi que par le mariage secret (janvier 1632) avec Marguerite de Lorraine que le roi rejeta.

Toujours à la recherche d'un statut honorable, Monsieur lia son action aux ambitions politiques de son gouverneur d'Ornano et des nobles tels Chalais, les Guise et les d'Elbeuf. Dans sa Maison, descendant en partie des Valois (Gatulle), il s'entoura de fidèles comme le duc de Bellegarde, le sieur puis duc de Puylaurens et le magistrat Le Coigneux, tous prêts à jouer un rôle politique majeur qui aurait ruiné les desseins du roi et du gouvernement.

L'implication de Richelieu dans la marginalisation de Monsieur fut égale à celle du roi et de la reine mère. À la nuance près que le ministre, soucieux de sa place et inquiet de la santé du roi, ménagea parfois Gaston, comme en 1628 lorsqu'il chercha à lui faire attribuer le commandement de l'armée d'Italie, proposition rejetée par Louis XIII. Le cardinal compliqua d'autant plus leurs rapports qu'il procédait par élimination progressive de ses ennemis politiques, comme avec Ornano et Chalais proches de Luynes, ou les Marillac proches de Marie de Médicis. Il faut dire que le roi opéra en 1630 un revirement d'attitude à l'égard de son frère et le nomma, malgré le refus de sa mère, lieutenant-général de la capitale pendant son voyage en Italie. Suite à cette décision du roi, Monsieur travailla avec son frère à la stabilisation de la monarchie. Peu avant le Grand Orage, Louis XIII prit l'initiative de réconcilier Richelieu et Gaston afin de ménager son frère. Le cardinal, quant à lui, s'était rapproché des conseillers de Gaston, Le Coigneux et Puylaurens, auxquels il promit des charges considérables, la pourpre pour l'un et un titre de duc pour l'autre. Le résultat fut que Monsieur, soit par intérêt soit par mégarde, ne se solidarisa pas avec Marie de Médicis durant la Journée des Dupes. Il ne changea d'attitude qu'en janvier 1631, lorsqu'il prit conscience que, seul opposant, il pouvait jouer un rôle central dans la crise.

\section{La place du roi, et le respect qui lui est dû}

Le roi lui-même joue un rôle moteur, contrairement à ce que nous disent et la vulgate historiographique et Morgues lui-même, qui convergent dans l'idée que le roi serait subordonné au cardinal - idée intenable aussi bien d'un point de vue hiérarchique que politique.

39 Au début de l'année 1631, une médiation avec Monsieur semblait encore possible, même après sa rupture de fin janvier. Dans ses missives des 23 et 26 février et des 10 et 26 mars, Louis XIII abordait la question épineuse des mauvais conseillers de son frère 
qui, selon lui, l'avait poussé hors de la cour. Il y défendait en outre l'action de Richelieu et déclarait sa volonté de "contenter " son frère, exprimant le "déplaisir » de leur séparation, qu'il assortissait de sa prière - » je ne laisse de vous en conjurer encore » de revenir auprès de lui ${ }^{14}$. Le ton changea à la fin du mois de mars suite à la sortie du royaume de Gaston (le 28 mars) et l'irritation qu'elle engendra chez Louis XIII. Début avril, la Lettre de Monsieur au roi publiée avec la réponse du souverain, puis, toujours du côté du roi, la Relation de ce qui s'est passé durant le séjour du roi à Dijon allumèrent la discorde.

Du 8 au 10 avril, Richelieu avait publié la Lettre de Gaston en ajoutant des apostilles que Monsieur adressait au roi et au cardinal ${ }^{15}$. Ces griefs se retrouvent en partie dans la Remontrance et se résument à : la reine était libre de décider de ses mouvements ; le roi agissait pour conserver son autorité et arrêter les projets des mauvais conseillers; la conjuration de Chalais était rattachée à la révolte de Gaston; les attaques contre le cardinal étaient des calomnies; enfin, le roi était libre et bien informé de ce qui se passait dans son royaume.

41 Le ton monta encore d'un cran avec la Relation, également vers le 8 avril, où Louis XIII prit violemment à partie les membres de l'entourage de Monsieur et les fit condamner pour crime de lèse-majesté. Dans le même temps, il adressa à son frère une missive (8-10 avril) dans laquelle il déclara que la Lettre de Monsieur au roi constituait déjà un manifeste de révolte contre lui, tout en lui reprochant de s'appuyer sur des mauvais conseillers. Afin de bien lui faire entendre sa position, il y rejeta la thèse selon laquelle il était prisonnier de la parole du cardinal en arguant, d'un ton ferme: "Je vois les choses comme elles sont et non comme on voudroit me les faire paroître. Je ne ferme jamais les oreilles aux vérités... ».

Dans cette salve de publications, on peut faire l'hypothèse que la Remontrance intervient après la missive du roi à Monsieur, donc après le 8-10 avril ${ }^{16}$, c'est-à-dire à une phase relativement modérée du conflit. En fait de «respect » et de place hiérarchique, la Remontrance considère que Monsieur a manqué de respect au roi : "si vous ne vous rendez dans les respects que vous devez au Roi... » (Remontrance, p. 33, II, §18, unique occurrence de respect). Quand donc Morgues critique Richelieu de manquer de respect envers Monsieur, il déplace habilement la question, en ne disant pas un mot du manque de respect de Monsieur envers le roi.

La suite immédiate de l'histoire est celle d'une querelle qui s'envenime, surtout après la publication du manifeste de Monsieur à la fin du mois de mai. Un premier point d'arrêt se situe à l'automne. Dans la période courant du 23 février au 20 octobre 1631, c'est-àdire de la première lettre du roi à Monsieur jusqu'à l'ordonnance confirmant la condamnation de ce dernier et de la reine mère, le parti du roi s'imposa à ses adversaires ${ }^{17}$. Les Marillac étaient emprisonnés, le parti des dévots dispersé, Marie de Médicis et Gaston vaincus ou presque. Deux dates sont à retenir pour mesurer le tournant politique accompli : le 19 juillet 1631, Marie de Médicis franchit la frontière du royaume pour s'établir aux Pays-Bas; en août, Richelieu devint duc et pair par volonté de Louis XIII. D'une part, l'échec de la reine mère, d'autre part le succès du roi et de son ministre. Le coup d'État (le second après celui orchestré contre Concini) permettait à Louis XIII d'imposer son autorité pleine et entière sur la monarchie. Seul Gaston s'y soustrayait, il fallait œuvrer pour le faire revenir, et, au-delà, pour qu'il accepte l'autorité absolue du roi et de son ministre : cela prendra encore trois années de lutte acharnée au sein de la famille royale. 


\section{La place de Richelieu : le contenu politique de la Remontrance} conseillers de Monsieur et la conclut par une exhortation à se rallier au roi et à ses bons ministres: les compétences de ces derniers s'opposent in fine à l'incompétence des premiers. Sous couleur de raisonner Monsieur, l'auteur attaque ses conseillers et Marie de Médicis. Ce schéma général permet d'associer à six reprises l'opposition bon/ mauvais ministre à l'éloge du cardinal, en couvrant ainsi admirablement la tripartition du texte entre passé, présent et futur (p. 6, 14, 15, 27, 28, 40-41). De fait, le thème des conseillers ou ministres incapables de Gaston fait le lien entre la narration du passé (Ornano, Chalais, Monsigot) et celle du présent (Marillac, la reine mère, Le Coigneux). Monsieur dépendrait de son entourage au point d'en être la dupe, avec pour résultat inévitable ses nombreuses imperfections, notamment sa crédulité, sa faiblesse, son inconstance dans le passé (p. 15, 30, 21-22 = I, § 7, « vous croyez trop »; II, § 16, « piper, et vous »; I, §9, «Pensez-vous...»), mais aussi ses espérances vaines pour le présent (p. 28, II, § 15, « la vanité de vos espérances »). Tout cela passe par une falsification des données historiques: 1 . les apanages obtenus par Monsieur, présentés comme un cadeau, alors qu'ils furent le fruit de sa révolte lors de sa première sortie du royaume ; 2. l'abandon par Monsieur du siège de La Rochelle, dû en réalité à l'humiliation infligée au prince, abandon suggéré justement par ses conseillers au nom de sa dignité ; 3 . le refus du commandement de l'armée d'Italie, qui en réalité lui fut nié par le roi jaloux de sa primauté guerrière (p. 10-11, 19, $24=\mathrm{I}$, §5, « grand apanage »; § 9, « quitter le siège de la Rochelle »; § 11, « le commandement de l'Armée d'Italie »).

Par rapport à la question des ministres et conseillers, la III ${ }^{\mathrm{e}}$ partie de la Remontrance est particulièrement intéressante. En effet, elle réfute la thèse formulée par Monsieur dans sa Lettre de Monsieur au roi et relayée par l'opposition, selon laquelle le roi serait innocent, ignorant de la vérité dissimulée par son principal ministre, et en somme prisonnier de celui-ci. Libre, le roi aurait mis de l'ordre dans le conflit, s'il en avait été informé. Or nous connaissons la réponse publique de Louis XIII, reprise dans la II ${ }^{e}$ partie : le roi affirme la primauté du souverain et le rôle subordonné du cardinal, qui sert les intérêts de l'État (p. 28, § 15, « Vous voyez que le Roi... »). Dans la III ${ }^{e}$ partie, on trouve la théorie du ministériat et sa nécessité dans la monarchie, dont la formulation anticipe celle du Testament politique de Richelieu ${ }^{18}$. Surtout, il y est affirmé le statut légitime et juridiquement " ordinaire » du ministériat ainsi que ses hauts résultats en France et en Europe. Richelieu-Sirmond fait donc comme si le ministériat allait de soi, alors que ce n'est nullement le cas. Il s'agit de légitimer la place de Richelieu, qui est dans les mains du roi, et non l'inverse.

En réalité, le ministériat est un outil politique transitoire : Mazarin en préparera la fin et Louis XIV le rejettera comme une déviation de l'ordre naturel de la monarchie absolue, comme nous l'avons prouvé récemment ${ }^{19}$. Quant aux mauvais conseillers de Gaston d'Orléans, au-delà de leur « cupidité » ou plutôt de leurs ambitions de carrière, les charges colossales et l'immense fortune cumulée par Richelieu prouvent mal que ce dernier travaillât pour la « gloire » du roi, comme le suggère la Remontrance (p. 9, I, § 4, 
«pour avancer leur gloire »). Les recherches de Caroline Maillet-Rao ont montré que le groupe lié à Monsieur et à la reine mère était aussi absolutiste et « impérialiste » que le cardinal : leur opposition au ministériat venait en effet du rejet de la délégation formelle du pouvoir au ministre, car ils prônaient la sacralité unique et absolue de la monarchie ${ }^{20}$.

Dans la Remontrance, les attaques sans « respect » de Richelieu à l'égard de Monsieur et de son entourage occultent donc le véritable enjeu politique de la Journée des Dupes : la conquête du monopole de la faveur royale. Dans cette crise politique majeure, l'instabilité des places de Richelieu comme de Monsieur tient à une cause unique. Louis XIII, loin d'être le jouet de son ministre, est le maître du jeu, un stratège habile, un roi secret, obscur, puissant, en un mot 'tacitien'. À cet égard, le faux exemple avancé par la Remontrance d'un Henri IV déléguant son pouvoir aux ministres (p. 35, III, § 20 , "Le défunt Roi... ») fera sourire le lecteur averti.

\section{Analyse rhétorique : « intention » et amitié}

Notre première partie a rendu compte de ce que Morgues perçoit comme un manque de « respect » envers Monsieur. Nous en venons maintenant à l'autre règle qu'il assigne à toute remontrance, l'«intention» d'être utile au destinataire. Cette règle-là est parfaitement valide, mais la lecture des traités de rhétorique va nous permettre de comprendre où se situe le sentiment d'irrégularité que Morgues éprouve, ou feint d'éprouver. Si toute remontrance a une intention d'utilité, un but d'"instruction", toutes ne sont pas nécessairement respectueuses du destinataire. L'«humble remontrance» est certes le cas le plus fréquent, mais - et Morgues le sait pertinemment - il peut $\mathrm{y}$ avoir des remontrances au ton très violent, loin de tout respect de la supériorité hiérarchique du destinataire.

Dans les traités rhétoriques de l'époque, l'intention se dit en termes d'" amitié », selon un sens de ce mot qu'il nous faudra préciser. Mais pour y venir, il faut se poser une question préalable, et fondamentale : de quel type de discours relève la Remontrance à Monsieur? Une telle question ne se poserait pas si le mot de remontrance renvoyait de façon stable à un type unique de discours. Il n'en est rien. Robert Estienne traduit ainsi remonstrer par obiurgare, admonere et spem impellere ${ }^{21}$, soit trois types distincts: objurgatio, monitio et hortatio.

Les traités énuméraient une vingtaine de types de discours, répartis selon les trois genres de la rhétorique: judiciaire, épidictique et délibératif, qui relèvent respectivement du passé, du présent et de l'avenir (Aristote, Rhétorique, I, 3). Dans la Rhétorique abrégée de Vossius (1621), le judicaire comprend cinq types, à commencer par l'invectiva et l'objurgatio; le délibératif en compte huit, dont monitio et hortatio. Non contents d'être distincts, les trois types d'Estienne n'appartiennent donc même pas à un seul genre : "judiciaire ", l'objurgation a un ton accusatoire ; "délibératives », la monitio et l'exhortation parlent d'avenir.

La Remontrance peut être interprétée de trois façons : invective ; objurgation; monitio. Une telle instabilité générique répond précisément à l'instabilité politique que nous venons de voir. 


\section{L'invective}


différence hiérarchique. Or, amicus chez Vossius se place sur un autre plan, celui de l'intention de l'orateur. La réprimande du général au soldat évite de conduire celui-ci à la révolte ouverte; à cela près, le discours peut être d'une violence inouïe. Nous reconnaissons la situation de Louis XIII face à Monsieur. Le roi, comme on dit, 'vient en paix', mais avec des propos aussi violents qu'une déclaration de guerre. Il n'est pas le général face au soldat, mais le "père envers son fils" de Vossius : le roi «vous veut traiter en frère et comme fils » (Remontrance, p. 5, exorde, § 1 ). Cette remarque est l'un des signaux qui disent l'intention: signaux très attendus par l'auditeur, et explicités dans les traités. La rhétorique rejoint ici les juristes de l'époque, pour qui le roi était le père de son peuple, et donc celui de son frère et sujet.

Morgues ne relève pas, évidemment, la formule sur frère et fils, mais c'est elle qui donne sens à sa remarque sur l'exorde. Quand il feint de s'offusquer que, avec un personnage comme Monsieur, on emploie aussitôt un mot outrancier comme monstre, il récuse en fait la prétention de la Remontrance à être une objurgation, et il la ramène à n'être qu'une invective, foncièrement hostile, belliqueuse.

L'objurgation est le paradoxe d'une invective 'amicale', ou en l'occurrence 'paternelle' : le père réprimande, mais 'pour le bien' du fils et de la famille (ou du royaume). De façon régulière, seul Louis XIII pourrait tenir une objurgation à Monsieur, et lui dire: « Retournez », mais « sans traiter » (Remontrance, p. 41, V, § 24). Revenez d'exil, vivons en paix ; mais sans conditions, car je suis votre supérieur, et donc en droit de vous parler rudement, de ne faire aucune concession.

\section{Monitio et avenir : la primauté du délibératif}

Qu'en est-il maintenant du rappel du passé ? Morgues a raison : la première partie de la Remontrance est une longue narration des erreurs de Gaston, avec pour incipit « Retournez-vous, et regardez le passé » (p. 8, I, § 4). La question est de savoir comment on interprète ce retour sur soi. Un rappel du passé ne se fait pas nécessairement sur un ton d'accusation. Ici, le retour sur soi prépare le retour en France : la visée est l'avenir.

C'est de nouveau affaire d'intention : tout dépend de la finalité, qui donne le centre de gravité. La Remontrance n'emploie pas, comme Morgues, le mot de fautes, on peut même supposer qu'elle l'évite soigneusement. Non pas faute, mais erreur. Le passé est là comme mise en garde pour l'avenir, pour prévenir l'erreur suivante. Cette narration a, comme souvent, une valeur argumentative: chaque erreur est une preuve que vous allez recommencer. Si donc le plus important est l'avenir, on est dans le délibératif, non le judiciaire ou l'épidictique (alors même que cette narration vire souvent au blâme). Ce qui est déterminant est de nouveau l'intention de l'orateur, son but: malgré nos différends, n'insultons pas l'avenir.

À l'intérieur du délibératif, il ne reste plus qu'à déterminer, parmi les huit types de discours qui en relèvent, celui qui correspond à la Remontrance. Ce ne peut être que la monitio, " admonestation, avertissement », ou tout simplement " conseil ». Morgues luimême le reconnait, pour le récuser: "Mais qui estes vous, qui auez l'effronterie de nous conseiller? »(p.81, ses italiques). La preuve interne est, comme d'habitude, la propositio. On la trouve à sa place, c'est-à-dire au début et à la fin de la Remontrance : bien visible à la fin, "Retournez sans traiter, et touché du Ciel venez-vous remettre dans les bras du Roi » (p. 41, V, § 24), et déjà dans le premier paragraphe, « votre retour 
et [...] la paix » (p. 5, exorde, $\S 1)^{25}$. Votre retour sera, au futur, le retour de la concorde et de la paix.

Qui parle ici, qui énonce ce conseil de revenir? C'est le roi : « écout[ez] le Conseil du Roi, qui vous conjure de retourner il y a si longtemps » (p. 38, IV, § 21). Il faudrait sans doute écrire aujourd'hui, sans majuscule, "le conseil du roi». Mais la majuscule emblématise notre problème. Dans la Remontrance, c'est le roi qui parle et/ou son Conseil, toute l'instabilité générique tient à ce et/ou. Le Conseil, ou le conseiller, ou le ministre, est et n'est pas le roi. Parce qu'il s'appuie sur l'autorité royale, il peut faire entendre le ton rude de l'objurgation hiérarchique. Mais parce qu'il n'est pas le roi, il parle un ton plus bas, en simple 'ami', au sens général de 'non hostile'. Ce mélange des voix correspond à la monitio "mêlée à de l'objurgation » que décrit Vossius dans un traité antérieur, avec un plan qui s'applique assez bien à notre texte ${ }^{26}$. Cela correspond aussi à la simple différence de degré que voyait Érasme entre admonestation et objurgation, la première étant relativement plus douce et aimable que la seconde, laquelle est plus libre de parole ${ }^{27}$.

Tout cela découle du traité de Plutarque, Comment on peut distinguer le flatteur de l'ami, référence obligée de l'époque. Vossius le cite aussi bien à propos de la monitio que de l'objurgatio, et sa thématique est très présente dans la Remontrance. Plutarque crée le mythe puissant du vrai ami qui se doit d'être franc, de critiquer librement, 'pour le bien' du destinataire. C'est beau comme l'antique, même si, dans la réalité, une telle franchise provoque souvent la rupture qu'elle a pour intention d'éviter. Une monitio est ainsi, selon une formule devenue ironique, le conseil d'un ami qui nous veut du bien. Un titre de 1644 le dit en latin : Amico-critica monitio ${ }^{28}$. Morgues le dit en français avec son propre titre : un ami sincère est un "Caton » qui donne un conseil «charitable ", manière chrétienne de dire « amical ».

\section{Conclusion}

70 La forme rhétorique de la Remontrance rejoint ainsi le fond, sa légitimation du ministériat, sa basse continue sur la juste place des ministres, ces " conseillers d'État " qui donnent des conseils ou avis nommés des « remontrances ${ }^{29}$ ». Car celui qui fait une monitio se nomme en latin un monitor. Chez Salluste, le monitor est le conseiller opérationnel que s'adjoint un noble incompétent mais élu général en chef, imperator ${ }^{30}$. En anglais du début du Xvir ${ }^{e}$ siècle, monitor désigne le conseiller, l'instigateur ${ }^{31}$. Ce «moniteur" est très proche du problème que pose l'idée même de ministre. La différence entre le bon et le mauvais conseiller/ministre est la problématique même du traité de Plutarque, en particulier de sa fin sur la liberté de parole (la parrhèsia), qui doit être "prophylactique » et non "thérapeutique »: donc tournée vers l'avenir, comme l'est « ce Discours tout plein de liberté » (p. 3, exorde, § 1).

71 Pareille liberté d'expression choque Morgues, qui renvoie au monde idéal où les remontrances régulières parleraient pour le « profit » et « instruction » du destinataire, mais 'humblement', c'est-à-dire en respectant les places dans la hiérarchie. L'inférieur s'adresserait au supérieur en mettant les formes (rhétoriques), sans mélanger les genres, sans emprunter la voix du roi. La Remontrance à Monsieur, texte de crise, est aussi la crise de cette régularité. La liberté d'expression ici à l'œuvre n'est pas celle des Modernes. Il s'agit de parler à un supérieur (Monsieur) en s'abritant derrière le supérieur de celui-ci (le roi). Ce mélange des voix irrégulier donne à Richelieu toute 
liberté de faire entendre deux choses à la fois : il préserve les apparences d'un dialogue, dont pourtant il ne veut pas dans l'immédiat, comme l'a fort bien perçu Morgues.

\section{NOTES}

1. Mathieu de Morgues, Charitable Remonstrance de Caton chrestien à [...] Richelieu sur ses actions, et quatre libelles diffamatoires, faicts par luy ou ses escriuains, s. l., 1631 (B. M. Lyon, 325274, visible sur Google), p. 41 : «Vous commencez les histoires du temps passé, par celles que vous dites que le Mareschal d'Ornano (que vous nommez Colonel) a fait » (en manchette : « Pag. 9. »).

2. La narration étant définie comme l'exposition du fait, elle peut être exposition des faits passés, présents ou même futurs (Quintilien, Institution oratoire, IV, 2,3); en tout état de cause, le plan suit l'ordre chronologique. Pour Morgues, la transition entre les parties I et II se situe sans doute un peu après, au début de notre $\$ 14$. Ibid., p. 81 (les italiques sont de Morgues, ici et dans les notes suivantes): «Vous concluez vostre dénombrement des fautes que Monsieur a faites par l'aduis de son Conseil, en disant, Voila de iolis Conseillers d'Estat!»; et p. 82 : «Vous quittez la poursuite des Ministres de Monsieur, et n'abandonnez point celle de la Reine Mere : vous ne vous lassez point de dire du mal de celle qui ne s'est point lassée de vous faire du bien. Vous dites, qu'elle a asseuré les Ministres de Monsieur, par Messieurs de Marillac, de vostre perte " (en manchette : « Pag. $28 »)$.

3. Morgues, ibid., p. 89 : « Vous venez sur la fin à vostre apologie ; vous taschez de monstrer, qu'il n'y a point d'apparence que vous entrepreniez contre le Roy et l'Estat, pour auoir quelques gouuernements et quelques charges [...]. »

4. Morgues, ibid., p. 96 : « Le reste de vostre escrit et de vostre esprit, s'employe pour representer à Monsieur sa foiblesse, et les forces de la France [...]. » (en manchette : « Pag. 37. 38. 39. »).

5. Morgues, ibid., p. 99: "Vous exhortez Monsieur de retourner sans traiter. Il ne le fera pas par vostre conseil [...]. »

6. S. 1., 1631, 196 p., B. M. Lyon, 325274 (sur Google). La réfutation y est aux p. 34-101 (voir aussi p. 29) ; elle semble avoir échappé aux historiens jusqu'ici.

7. M. Deloche, Autour de la plume de Richelieu, Paris, Société française d'imprimerie et de librairie, 1920, p. 464-466.

8. Par exemple, BnF, 8-LB ${ }^{36}-2822$. Les B. M. de Versailles, Lyon, Reims, Nantes, Albi, etc. en conservent chacune un. Peut-être eut-elle le temps de paraître une seconde fois en 1631, pour faire suite à la Lettre de la Roine mere au Roy, s. 1., s. d. [1631 ?], BnF, 8-LB ${ }^{36}-2838$. Enfin, si le CCFr signale à tort une copie manuscrite à la BnF (Fonds Dupuy n ${ }^{\circ} 569$ ), il en existe une à la B. M. de Tours (ms. 1062, f. 88-96).

9. Médiathèque de Troyes, fonds ancien hh.9.3818 vol. 7 (27).

10. «Les pamphlets de Mathieu de Morgues », Revue française d'histoire du livre (RFHL), 1978, $n^{\circ} 18$, p. 41-86.

11. Petits chiens de compagnie. Voir « mignon de couchette » (Littré, Dictionnaire [...], s. v. mignon $4^{\mathrm{e}}$ et couchette $2^{\mathrm{e}}$ ).

12. Advertissement de Nicocleon à Cleonville [...], s. 1., 1632, cité par M. Deloche, Autour de la plume de Richelieu, op. cit., p. 456-457.

13. J.-M. Constant, Gaston d'Orléans prince de la liberté, Paris, Perrin, 2013 ; P. Gatulle, Gaston d'Orléans entre mécénat et impatience du pouvoir, Seyssel, Champ Vallon, 2012. 
14. Voir respectivement P. Grillon, Les papiers de Richelieu, Paris, Pédone, VI, 1985, p. 102 ; mêmes mots dans la lettre du 23 mars, ibid., p. 149; L.-M. Avenel, Lettres [...] de Richelieu, Paris, Imprimerie Nationale, IV, 1861, p. 107, 109. - Dans la Remontrance, seul le roi « conjure » Monsieur (p. 13 et p. 38 = I, § 6 et IV, § 21).

15. Voir P. Grillon, Les papiers de Richelieu, op. cit., p. 200-207, 217-220; L.-M. Avenel, Lettres de Richelieu, op. cit., p. 125-130, 130-132, 153-170.

16. Le terminus a quo est le 6 avril, où sont signés les accords de Cherasco : « nous sommes en paix avec l'Espagne et l'Empereur » (Remontrance, p. 39, IV, § 22).

17. Il s'agit d'une dizaine de documents imprimés en 1631 et publiés ensuite dans le Mercure françois, t. XVII, 1633, p. 118-390. Certains parurent aussi dans Paul Hay du Chastelet, Recueil de diverses pièces pour servir à l'histoire, s. 1., 1635, passim; on les trouve encore dans les recueils scientifiques suivants : L.-M. Avenel, Lettres de Richelieu, op. cit., p. 96-236 ; P. Grillon, Les papiers de Richelieu, op. cit., p. 102-226.

18. $\mathrm{I}^{\mathrm{re}}$ partie, chap. 8, éd. F. Hildesheimer, Paris, Société de l'histoire de France, 1995, p. 212.

19. Voir G. Ferretti, «Mazarin, Louis XIV et la liquidation du Ministériat », I. Loskoutoff (dir.), Mazarin, Rome et l'Italie, sous presse.

20. C. Maillet-Rao, La Pensée politique des dévots, Paris, Honoré Champion, 2015.

21. Dictionaire Francoislatin [...], Paris, R. Estienne, 1539, s. v. Remonstrer. Remonstrance (avec aussi suadere). Sur les types de discours, voir Fr. Goyet, "Le problème de la typologie des discours ", Exercices de rhétorique, 1| 2013 (http://rhetorique.revues.org/122). Pour une application à des discours effectivement tenus, voir G.Ferretti, "Diplomatie et rhétorique. Les discours d'ambassadeur, genre et pratiques ", dans S. Andretta, L. Bély, A. Koller, G. Poumarède (dir.), Expérience et diplomatie, Savoirs, pratiques culturelles et action diplomatique à l'époque moderne ( $X V^{e}$ XVIII ${ }^{e}$ s.), Rome, Viella, 2020, p. 259-274 : p. 264-265, discours de remerciement ou gratulatio tenu à Charles IX par divers ambassadeurs pour le remercier de l'édit de Saint-Germain en 1570 ; p. 265, demande de soutien ou petitio adressée aux Cantons suisses en 1632 ; et, de façon plus développée, p. 265-274, demande de pardon ou deprecatio de Gênes à Louis XIV en 1685.

22. Gerard J. Vossius, Rhetorices contractae sive Partitionum oratoriarum libri quinque (1621), II, 26, $\S 1$, Leipzig, Kirchner, 1660, p. 214 (nous traduisons, ici et ensuite).

23. Ibid., p. 216 ; trad. Ph. Collé en séminaire RARE (Université Grenoble Alpes).

24. Avec renvoi à la p. 42 de la Remontrance (autre état du texte ?), laquelle a seulement (p. 29) : « vous ne deviez pas ensuite jurer amitié au Cardinal » (ce que Morgues commente, p. 84 : «Vous traictez comme egal auec Monsieur »).

25. Voir encore « si vous ne retournez ", clausule de la troisième partie (p. 36, III, § 20).

26. Commentariorum rhetoricorum, sive Oratoriarum Institutionum libri sex [1605], Leyde, J. Maire, 1630, III, 7, ch. 2 De Monitione, p. 392, sur « une autre méthode d'admonestation, jointe à une objurgation [monendi rationem itidem objurgationi conjunctam]. » Elle comporte « une narration complète de toute l'affaire » et une fin où «non seulement nous le détournerons de ce défaut en le terrifiant, mais encore nous l'exhorterons à vivre conformément au bien [non à vitio modò deterrebimus, sed etiam adhortabimur ad bene vivendum]. Car en général l'admonestation et l'exhortation vont ensemble.»

27. "Blandius" vs "liberius", à propos de la lettre monitoria adressée à un supérieur : De conscribendis epistolis, éd. J.-C. Margolin, dans Opera Omnia I-II, Amsterdam, North-Holland, 1971, p. 488-489 (LB I 445).

28. Anonyme [Claude de Mesmes], Francfort-sur-le-Main, s. n.; réfutée par Morgues en 1645 (Amico-Criticae Monitionis Litura Franco-Galli Calamo Ducta, Paris, s. n.).

29. Unique occurrence (p. 27, II, § 14).

30. Jugurtha, 85, 10 (discours de Marius au peuple) : "monitorem officii sui ». Chez Cicéron, le monitor est en-dessous du maître ou magister (De Oratore, II, 99). 
31. Parfois en mauvaise part: "When a man is author and monitor to another to commit an unlawful act » (Francis Bacon, The Elements of the Common Lawes of England, Londres, More, 1630, p. 66).

\section{AUTEURS}

\section{GIULIANO FERRETI}

UMR 5216 Litt\&Arts (CNRS / Université Grenoble Alpes FRANCIS GOYET

UMR 5216 Litt\&Arts (CNRS / Université Grenoble Alpes 